\title{
Bio-Inspired Computing-A Dive into Critical Problems, Potential Architecture and Techniques
}

\author{
Ajay Sudhir Bale ${ }^{1}$, Subhashish Tiwari ${ }^{2, *}$, Aditya Khatokar ${ }^{1}$, \\ Vinay $\mathbf{N}^{1}$ and Kiran Mohan $\mathbf{M} \mathbf{S}^{3}$ \\ ${ }^{I}$ Department of ECE, School of Engineering and Technology, CMR University, Bengaluru 562149, India \\ ${ }^{2}$ Department of ECE, Vignan's Foundation for Science, Technology \& Research, Vadlamudi, \\ Guntur 522213, India \\ ${ }^{3}$ Department of CSE, School of Engineering and Technology, CMR University, Bengaluru 562149, India
}

("Corresponding author's e-mail: Drst_ece@vignan.ac.in)

Received: 26 March 2021, Revised: 9 June 2021, Accepted: 15 June 2021

\begin{abstract}
The integration and development of electronics in the recent years have impacted a major development on the world and humans, one among that is nanotechnology. Nanotechnology has achieved a greater progress in biomedical engineering in diagnosis and treatment, leading to the introduction of nanomaterials for drug delivery, prostheses and implanting. This work describes the Bio-Nano-tools that are developed based on iron oxide properties, automated tools used in the tumor detection, satin bowerbird optimization (SBO) technique employed in diagnosis of skin cancer. This work also highlights the post introduction development of nanomaterials like combination of nanotechnology with Artificial Intelligence (AI) and its impact, advancement of nanomaterials based on their operations, shapes and characteristics that leading to the growth of nanostructures with operations control properties. The paper also highlights the improvement of silicon neuromorphic photonic processors and parallel simulators in the development of bio inspired computing. We are hopeful that this review article provides future directions in Bio-Inspired Computing.
\end{abstract}

Keywords: Silicon neuromorphic photonic processors, SBO, Nanomaterials, Drug delivery, Efficiency, Optimization

\section{Introduction}

The understanding of brain has led to greater developments in the past century [1]. This has led to development of the electronic hardware which can study the neural system. Based on the development of neuroscience, several techniques have been developed to build chips such as spike encoders [1]. The advance in algorithms has brought in the revolution of AI. The demand to IoT is increasing, this is making the neural network algorithms complex. The Machine Learning (ML) along with metaheuristic [2] provides optimal solution which can have higher number of combinational optimization problems (COP) in short computational time. Metaheuristic refers to the algorithms which helps in solving complex problems where-in each problem is not adapted to them. Some of the algorithms are inspired with the features in nature. This nature inspired will have stochastic components, with requiring the parameters to be fined-tuned [2,3]. The popular algorithms [2,4-13] are shown in Figure 1. 


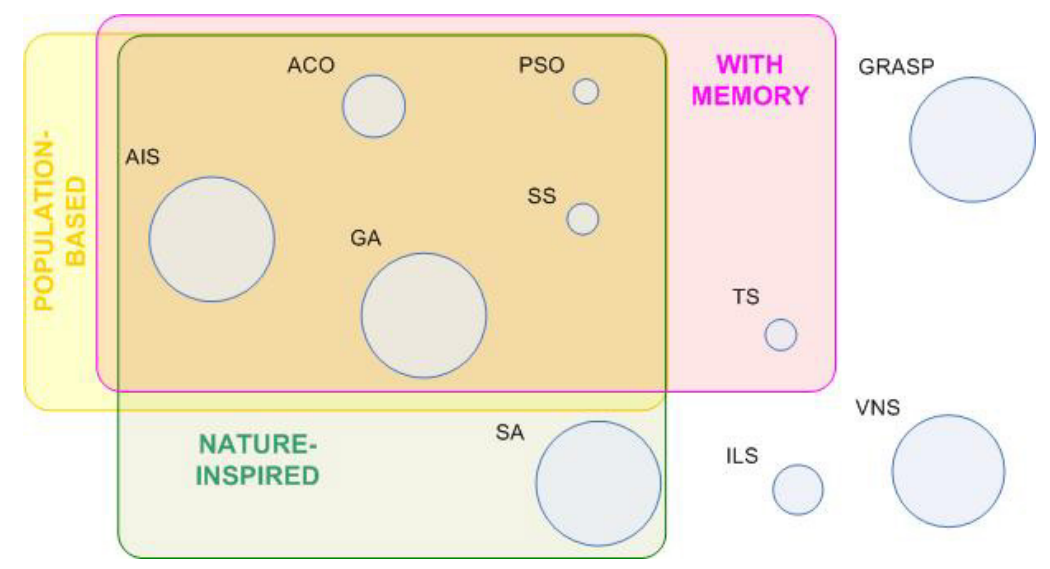

Figure 1 Popular metaheuristic algorithms listed as per different criteria [2].

The classification of various metaheuristic as in [3] is listed as per the different criteria. The $1^{\text {st }}$ criteria is based on population, $2^{\text {nd }}$ criteria is based on usage of memory and the last criteria is about their nature inspired features.

The performance of various techniques in ML is increased by the contribution of metaheuristic. The advantages of using ML algorithms with metaheuristic is depicted in Figure 2.

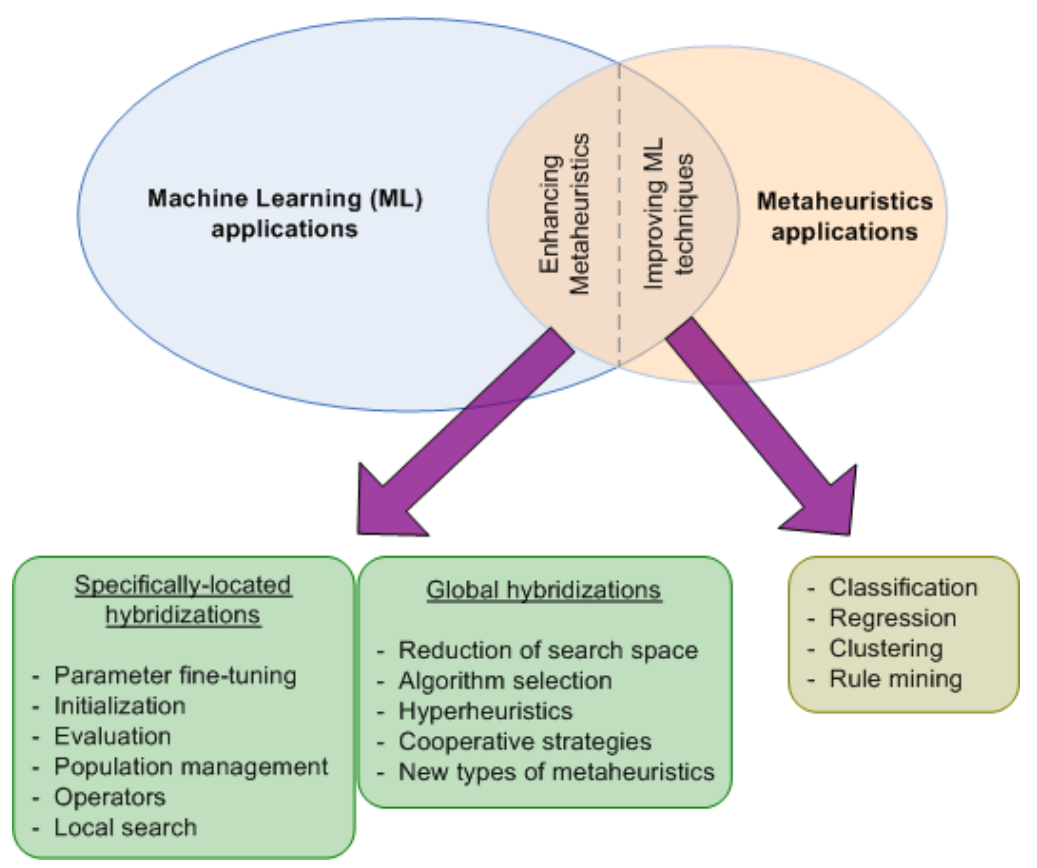

Figure 2 The advantages of using the combination of metaheuristic algorithms and ML [2].

\section{Soft computing techniques}

Nanotechnology has made a remarkable progress in the recent years. There are several interpretations and applications of nanotechnology, but all of them highlights development and design of bottom-up high order nanostructures, that re exposed to stimuli to give a particular response [14]. The nanomaterial applications are tuned by physics and surface chemistry. Moderating the nanomaterial surface in various ways can generate materials having discrete biological properties for particular end applications and also physiological conditions with increased solubility $[15,16]$. The advancement of nanotechnology in the field of biotechnology had introduced the hybrid science called as Nano 
biotechnology [14]. The Nano biotechnology evolved its applications in drug deliver [17], diagnosis, implanting and prostheses.

Utilizing physical and chemical techniques and using complex biological reactions, like hybridization of DNA-DNA antibody- antigen and receptor- ligand interactions, leads to bio specific molecular conjugation with nanoparticles. Particular applications of nanomaterials with their relevant toxic effects are determined by surface physics [18,19], surface chemistry [20,21] and surface thermodynamics [22].

The nanoparticles of metal oxide are used for building many medical devices. The tools for diagnostic and therapeutic purposes are developed based on the iron oxide magnetic properties, like MRI (Magnetic resonance imaging), ultrasonic technology [23] and magnetic particle imaging, magnetic particle hyperthermia [24]. In the recent years metal oxide nanoparticles are utilized in many biomedical applications because of the high surface area and improved magnetic and catalytic operation [25,26]. Titania (TiO2), Ceria $(\mathrm{CeO} 2)$, Zirconia $(\mathrm{ZrO} 2)$ and Magnetic iron oxide (Fe3O4) are most commonly used nanoparticles. These components have significant behaviors in bactericidal, antioxidant and catalytic activities, biocompatibility, and mechanical stability which makes them ideal for number of different biomedical applications, like bio imaging and therapeutic agents, medical implanting, drug delivery systems and neurochemical tracking probes [27-31].

Skin diseases are a form of cancer from which the skin tissues, tumor cells are created. Skin disease, nevertheless, is a harmful condition, but effective diagnosis of such an illness allows health professionals to treat it [32]. An automated machine system for the initial detection of tumors is introduced in the current report [32]. A modern image classification focused on the convolutionary neural network designed through modelling had been introduced since noise reduction dependent on a median gradient during the initial phase as well as its performance was suggested by validation set [32]. Skin disease is an elevated condition that happens in the predominant portion of the skin. Skin disease typically occurs on the surface of a skin, which would be present as inflammation, bumps, or even other areas of the skin at first [32]. In addition, therapists or radiologists are assisted by the importance of image analysis in therapeutic diagnostics to minimize uncertainty as well as improve the performance of primary prevention for medical diagnostics. Algorithms are among the advantageous instruments for detection of therapeutic tumors. A convolutionary neural network is focused upon deep learning that is often utilized where many levels are educated in innovative ways [33-35]. Such systems are some types of computational layered framework developed for image-like, 2-dimensional information [32].

Various sections of an object, that are centralized, were introduced as contributions to a neural net added and the solution is obtained by implementing new filtering [36,37]. Utilizing array of diverse, convolutional layer concatenates the image. There are many repetitive manufacturing throughout the structure which convolves the feature vector of filtering which output parameters could be educated. Over image, such modifiers shift. Each collection of functionality is generated by increasing independent application, which eventually gives one use of proposed feature moderators. The system becomes synthesized during preparation, as well as the information gathered from neural network is calculated with quite an amount which we assume is indeed a prejudice value as well as processed throughout the attribute field. In general, the gradient descent is preceded by neural network, which are used to minimize the size of functionality or requirements of a system. Once collected being transferred around, diagnostic photographs are sometimes impaired by interference. The objective of the techniques for object noise reduction is to remove certain noise that image's critical characteristics as often as necessary. Dermoscopic imagery is a commonly used therapeutic imaging method, since it is cost-effective, reasonably reliable, yet resilient. Target interference is indeed a failure, while it damages the accuracy of photographs or impacts individual activities of perception and identification. As a result, level refinement is a crucial step for both the processing, examining as well as understanding tissue samples of the feature [32].

The technique towards collecting further detailed data from the tumor region is termed attribute removal. In this stage, the aim of attribute extraction was to utilize the pattern as a tool for detecting skin cancer by separating the field of skin cancer. An actual image is indeed a random period with quite a large amount of details. Such clarification complicates the process in image analysis which takes time. For optimizing the operation, a better technique herein would be to use function removal. All reference images were denoised based on a median filters for achieving greater precision for analysis. In optimized approach centered mostly on method of maximizing image segmentation or reducing the sequence of aspects were introduced to improve the image classification $[32,38,39]$.

The algorithm of SBO is a modern meta-heuristics algorithm that was proposed based on male bowerbird attracting females [40]. This SBO algorithm is proposed for Dermoscopic imaged based skin 
cancer diagnosis, where it uses the CNN technique to increase the accuracy of the network. As shown in Figure 3, the image quality is increased by median filtering and then the aforementioned optimized CNNbased image segmentation is applied. The further step attributes of the segmented area are extracted for simplify classification. After obtaining the required features from the image, classification of an image into cancerous and healthy ones is undergone through a support vector machine (SVM).

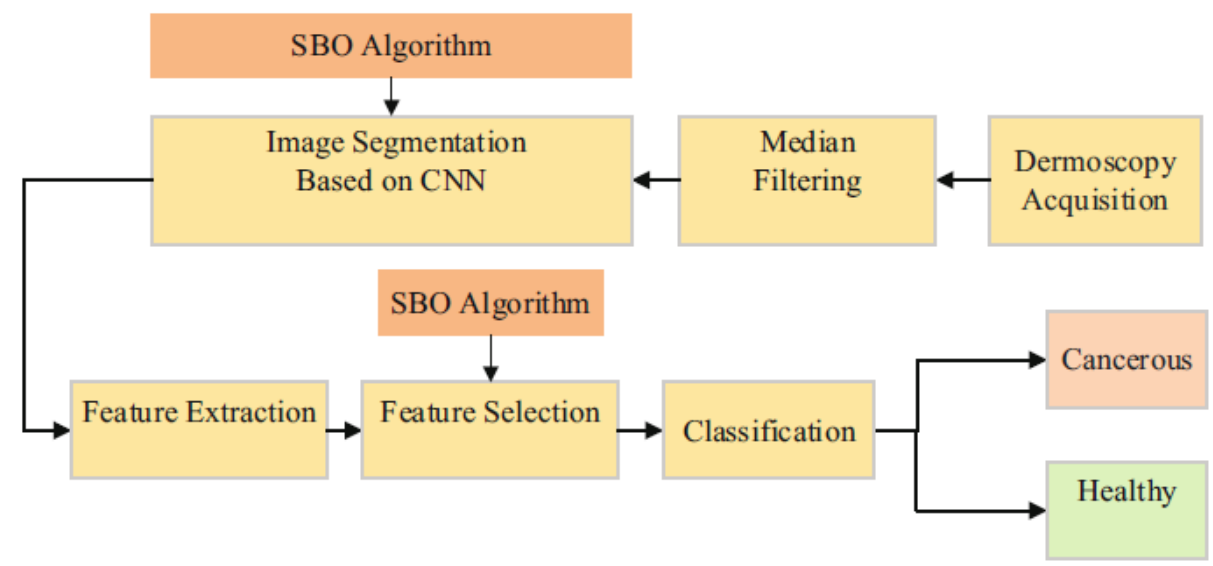

Figure 3 Process of SBO algorithm. Reprint with copyright permission from [32].

\section{Test case: Optimization and automatic generation}

Test case development is a tool for defining test information or following the requirements for application development. The development of test cases is a critical term being included in software development, and could be extracted from the specifications of application specifications [41]. An automated test scenario methodology immediately decides where the check proposed methodology produces the data sets and test results [42]. To ensure it accessible without glitches or faults and to enhance the consistency, the software development phase is a vital activity [43]. The calculation of user friendliness utilizes many criteria, like consistency, performance, and accessibility of code, extensibility, and many more. Application stability seems to be a more critical consideration in such consistency metrics since it tests how often code is reliable by accepting errors over the software lifespan. The code consistency concept is the efficient operation of the machine at a given period without any mistake. The requirement to forecast or approximate program performance utilizing modern methods including strategies in order to render the process quite accurate and perhaps less failure, reduced downtime. In a wide region of workforce at the relativity of several elements, it is a complicated challenge to determine the right benefit.

The automated test scenario creation mechanism implementation helps software development engineer but reduces additional time. However, with elimination in testing period, the expense of analysis reduces. To minimize the testing time or the volume of test events, a cluster analysis technique is used. One such approach gathers the same fragments of test scenarios that tend to identify repetitive test scenarios. To explain the full-fledged contingent covering but its pricey, this approach utilizes cyclomatic difficulty. Within the preliminary stage of the application design process, the accurate estimation of the application expense is quite difficult. The Deep Network-based paradigm is again a current form of efficient device testing and development strategy. Two types, such as the research climate or the operating condition, are known as external variables in software creation. After lowering the program expense, the neural Network based model methodology measures the duration span of an optimum software development or repair term $[44,45]$. Design research is a process which reflects the behavioral model which encrypts clear terminology as well as criteria for the success of the design. The design involves a set of entities expressed by parameters as well as the interaction between entities. The requirements for test exposure were determined by means of test instances covering a variety of items, as well as the production of test instances is decreased. In this cuckoo analysis process, the test scenario is provided as an entry to the composite process to evaluate all test scenarios received. In the cuckoo hunt, the test scenario is represented as the native species and an ideal test scenario is sought utilizing the Levy method [41]. 
The performance of the composite approach with a high execution time is the optimum test scenario. A provisional community is randomly produced or exercise parameters are displayed or retained for its accuracy. Estimations were made of the strongest preliminary optimum quantities. Upon this, on the basis of certain fitness characteristics, the design parameters were arranged. The highest performance attributes reflect the approaches that are closest to computational complexity. The lower halves of the bad approaches were discarded after the activity is planned or the higher quarter of the better approaches are used. This technique is being utilized to produce test scenarios that were configured through immediately having an instance of an ATM machine's exit process. Depending upon its exercise feature, test information variables are chosen. The suggested methodology tailored the test scenarios through minimal variations or period to optimize them [41].

\section{Nano engineering in biomedicine}

Modern advancements in bioengineering mainly rely mostly on growth of Nano engineering [46]. Since exposure to specific characteristics in nanomaterials is not easily accessible through conventional approaches, Nano engineering is an efficient method to design and technology that has also significantly enhanced or strengthened both the efficiency as well as functionality of nanomaterials. Over recent decades, advances in structural technology as well as advancements in research and technology have influenced the development of the nanotechnology field. Treatments or biologics, spanning across individual devices to medication creation and execution, were transformed, boosting numerous possible prospects throughout the treatment of challenging yet diverse illnesses [47-49]. It is observed that the potential to evaluate structural implementations with desirable characteristics as well as enhanced quality plays a significant part throughout the creation of futuristic structures that will offer innovative manufacturing long term possibilities in the domain of biomedicine. Nano engineering helps the modification for atomic as well as biological nanomaterials including its manufacture of nanostructures containing controlled operations [46].

The significance of Nano engineering is whether it could be used at the macroscopic level to locate the small or nanostructure indications concealed within substance results. Biomaterials' nanoscale structures typically hinge on functional arrangements or attitudes. The shapes, characteristics, as well as operations of nanostructured implants could be well defined as Nano engineering enables studies to concentrate on the transcriptional modifications in various substance frameworks or complex reactions to various events [50,51]. Amongst these popular biomedical-related resources, structural substances or composites are the key constituents of the individual anatomy or pharmaceutical equipment. In these frameworks only at molecular, ordered nanostructures and undifferentiated complexes give different interesting characteristics that lead to functional biomaterials. In comparison, nanomaterials typically have centralized architectures that translate the useful attributes of intermediate key components against the efficiency of subatomic materials. In recent times, its application of Nano engineering in diverse substance structures also advanced exponentially, opening up numerous prospects for both illness detection and diagnosis. Popular substances used in biomaterials, particularly composite alloys, nanoparticles or nanomaterials, were identified as well as its basic roles in the individual organism or in implantable implants were described [52,53]. Using Nano engineering methodology, the framework relation is understood and applied in such substances. The characteristics of the fundamental substances are highly dependent mostly on role or efficiency of implantable implants including biologics [46].

The most popular components used throughout biomaterials are nanomaterials, polymers, as well as biocompatible. In durability, elasticity, durability, resilience, as well as thermal conduction, different metals have several benefits that promote implementations in various industries, namely physiological functions, surgical implants, dentistry, as well as biosensors [46,54-59]. While exceptional microstructural and chemical characteristics result in economic use of composite alloys as key parts for bioinformatics use, modules, processing starts or bio - compatibility limits for specific criteria are indeed evident. In any substance witnessed in everyday situations, including functional microbes or growing organisms through microelectronic devices or constitutional structures, polymeric systems are generally included. In biomaterials like optics, transplantation, implantable devices, oral components, gene therapy, as well as regenerative medicine, these were also deeply engaged. In manipulating biodegradable polymers, Nano engineering plays a fundamental role by delivering excellent molecular range, accurate estimate of favorable characteristics, or effective feature guidance [46].

For biological organisms, polymeric materials become crucial. Nucleotides that hold genetic material, residues that cause metabolic transitions or fats that also offer stability as well as durability of the plasma membrane were common polymeric materials in living organisms. Nanomaterials were multistep substances comprising multiple state structures at only a manometer-order scale, offering 
special characteristics for specific thermal and physical characteristics. As stabilization for a molecular and polymer framework, nanomaterials consisting of specific substances and made of biomaterials were widely used. The varieties of nanostructured constituents or measurements have brought tremendous promise for major efficiency enhancements as well as the development of biomaterials. The interdependent architectures of nanoparticles, nevertheless, find it harder to specifically classify substrate activities or explain in depth the interfacial bonding between distinct process entities. The actual excellently supervised learning is still needed for refinement, considering the desirable success of Nano engineering configured with neural networks. Any surgical instruments, like unnatural skeletons or tumors, have been in direct communication for a number of years against areas of the individual system. In the simulation as well as development phases, while the introduction of AI through Nano engineering is already restricted, the supervised learning for AI is indeed inadequate with all substances and to fulfil certain specifications. Selection of major ones or the efficient implementation of AI strategies, quantity or reasonable quality management is vital [46].

The risk of nanomaterials comprising nanostructures stays uncertain, as potential harmful effects could not be explicitly reported by Nano engineering. While protection screening of biomaterials was already carried out prior to the submission, there is still inadequate evidence accessible mostly on adverse effects of nanoparticles on individual well-being. The viscosity or retention throughout the biological system of nanoparticles, particularly unsolvable nanomaterials that can also be absorbed and eroded, may ultimately cause tissue damage. Moreover, higher Nano-composites' density to volume ratios means enormous product reactions; in effect, this could cause certain unexplained biological processes that may change structural physicochemical characteristics. For the advancement of Nano engineering, ensuring precision of simulation of biomaterials, like material properties, composites or nanomaterials, is important. In recent times, Nano engineering, assisted by innovative AI techniques, is already being an effective method with a growing emphasis on biomaterials but has posed tremendous promise as a ground-breaking innovation within this area over the next decade [46].

The excessive proliferation in skin tissues owing to DNA degradation that couldn't be restored is known as melanoma [60]. Melanoma that arises through epithelial cells is among the toughest forms of melanoma. Whereas some forms of melanoma have reduced dissemination potential, the risk of cancer benefits in its capacity to spread very quickly. There is a significant explanation for these separation; another very harmful type of complexion disease is melanoma, but it is known to be the most fatal. While empirical research suggests that just $2 \%$ among all instances with tumors were melanoma, this contributes for $75 \%$ including all fatalities from tumors [61]. In epidermal layer, skin cancer begins. Unlike many other forms of melanoma, this could spread easily across other areas [62]. Via skin, systemic circulation or bloodstream, the tumor may mutate. When spreading across skin, this can disperse just to nearby neighborhoods; but if it reaches the lymph as well as connective tissue, it might propagate to other soft tissues. The skin cell which is infected by cancer becomes a tumor development that is hard to cope with $[63,64]$. Apparently, cancerous development develops mostly on cell membrane, specific address exceedingly likely, if diagnosed early, by a good reference examination as well as a total treatment. The approach towards effective treatment of skin cancer continues through group recognition. There are many numerous services and activities only at present which will measure epidermal layer defects or skin tags [60]. Skin cancer is a really serious tumor, but inspectors may clearly recognize it [60].

Due to the extreme principle of the "morality" dilemma, there seems to be a clear want of automatic evaluation metrics [60]. Such concern derives from various perspectives and stages of knowledge of researchers with tactile cancer. An illumination would then be used throughout the device that contributes to a sophisticated epidermal layer tumor magnification named the dermascope $[60,65,66]$. It was essentially a device that consists of a designed beam of light as well as lenses to analyze the epidermal layer at illumination ranges between $60 \times$ to $100 \times$ [60]. The optical observer puts the unit in between lenses as well as the tissue mostly on cell membrane with such a specific substance or creates a distinctly high - resolution image of the tumors or even the lump. Even "ambiguity" also plays a key role in communicating as a consequence. On what researchers have in object categories there is, thus as a consequence of a clear need for automatic detection equipment or help resources, as well as the issue in ambiguity of such widespread numbers. Melanoma is a tumor which is very harmful. This threat arises in its tendency to grow out of control; luckily, if detected in its initial stages, it is indeed treatable [60]. Regulations and also separate public services and activities for the evaluation of skin condition tumors including skin tags is already widely accessible. Current trends show that perhaps a system deep residual processing or fuzzy models can keep increasing the progress rate throughout this field of study [60]. 
There is no question that emerging problems and massive readily viewable statistical analysis will continue to accelerate such state-of-the-art accomplishments [60].

\section{Parallel simulators}

Parallel networks represent innovative computer structures in the Membrane Processing field, built on the basis or mechanisms that take part in living tissue [67]. Set of interconnected or complexity principles, and evolutionary modelling, are the most effective implementations of parallel systems. Provided that structures were just getting more difficult to work over, prototypes were also important resources for parallel models as well as its usefulness are crucial. Such systems get to consider a worst possibility does exist, but they seldom exist, in ability to respond with the different conditions which can emerge also during simulation. Technique used to control network is a computational model influenced by biological cell behavior or architecture. Such model also resulted in many types of highly parallel networked computers identified as dynamic frameworks or concurrent processes, essentially [68]. In a transformation stage, parallel processes adapt through 1 phase to another via a well before series of laws $[69,70]$. A processing of the machine is indeed a series of certain phases or architectures. The main aspect in structural implementations seems to be the vast with simultaneous parallel processing aspect: inside each substrate, challenges can be addressed in an optimally distributed mode, since all dynamics grow at network level in conjunction. In order to build evaluation, evaluation including simulated modelling methods, order to simulate parallel structures seems to be of vital significance. Status and trends parallel structures, for example, have been developed as well as effectively used for ecology modelling, but its effective modelling seems to be the secret to the method of rapid model creation, configuration of parameters and innovation. The generalized model architecture framework consists of several configurations: the description of a parallel framework to be modelled, the implementation with 1 or more simulations, as well as the compilation of design calculation performance results. The mechanism could also represent a defined parallel device's operation or instead delegate the definition to many other types that really can act as feedback to additional control systems. It may never be easy to incorporate parallel machine computation in advanced multiple computing, primarily because of its semi or concurrent design, however it has being demonstrated where parallel computing systems could be effectively used for this purpose. In some cases, like ecology modelling, standardized modules were required in order for prototype developers to use only single model with all different designs. Just a range of principles could be implemented within each framework. In general modules, this sort of incredible resolution will always be overlooked. The notion of resolving the distance between the developer of the framework as well as the creator of the device is discussed via the adaptive model method. It will allow programmers to define some linguistic elevated characteristics which will allow systems to adequately conform to the framework. Standard modules of the distributed connection are programmed to expect that worse conditions can exist, such as implementing all the specified principles at a specific instance. Normally, such versatility arrives at a reliability burden. Special modules, on the other hand, are intended for a training set confined to only single group of parallel applications $[67,69,70]$.

\section{Silicon neuromorphic photonic processors}

While addressing most of modern requirements of biological systems, nanotechnology devices have faced obstacles. Advanced deep neural networks have greatly impacted computational methods; however advanced electronic equipment was already established to execute these quite effectively [71]. The quantum incorporation technology with silicon intends to add computational physics to production environments currently designated for Nano electronics [72,73]. Easy optical process control specialties wherever devices do not even have adequate bandwidth or adaptability have indeed been identified by superconducting systems. The objective of computer vision Nano electronics is to transform theoretical frameworks of device applications structures to conceptual ML models. This introduced a different potential for sub-nanosecond timeline computer biological systems, including computational computing applications, sophisticated transceiver computer vision, and legitimate monitoring. The method of microelectronic architecture would be to compartmentalize, information services, the possibility of evolving theoretical principle. The indications whether Moore's law is changing are still there [71].

After all, only with advancement of modern technology or frameworks, like multi-core implementations, visual integer cores, including field-programmable gateway assemblies, nanotechnology has continued to achieve an increasing pace of progress [71]. Neural networks are all here to remain, but experimental computational techniques, evolving through microelectronic robotics to electromagnetic biomaterials, are seeing a revival in past years. There are no photonics with same character traits as microelectronics. It is indisputable it for increased transmission throughout long ranges, microelectronics, 
especially fiber electronics, is advantageous [71]. The goal of microelectronic photonic devices is to connect the country combined to know the implications which both frameworks may produce mostly on transmission of device knowledge. Neurobiology aim of recognizing perception or pathology-has inspired computer simulation of biological systems; it is been inspired by software development goal of creating better, most effective computers [71]. Such converging motives therefore resulted in converging studies through frameworks which strive to preserve a commitment to neuronal behavioral critical infrastructure which do not. As a core component of advanced AI underneath the pseudonym "natural language processing", deep neural networks have strengthened. In independent systems, AI is facing significant growth with increased competition. It is clearly prevalent whether traditional computational modelling really are not designed to incorporate performance and power consumption ML models which are appropriate for implementation requirements [71].

Modules of a deep neural network result in relatively basic spatial links connected by either a network consisting that can be configured. In functional data analysis and predictive modelling, like photonic computation including computerization, photonic integrated structures were suggested. These methods take account of the positive features of light waves: its fundamental consistency in insertion loss resonators; its elevated stability in vibrational networking sites; as well as their wide angle homogeneity. Evidently, semiconductor material satisfies the requirements for imaginary "photonic silicon," that has the ability to revitalize modern quantum computation inquiry. The Nano electronics group has gained significant recent interest through photonic reservoir computation approaches that benefit greatly from some neural characteristics. Such approaches were a providing a mechanism of the larger principle of reservoir computation focused on convolutional neural networks. The techniques to neuromorphic including reservoirs vary significantly and have productivity and higher. Via a vast range of physical permutations combined by vibrational factors of experience, all extract a vast variety of traits [71, 74-77].

In order to accommodate modern millimeter-wave channels as well as sophisticated multi antenna techniques, electromagnetic front-ends should change significantly. The principle of designing photonic systems or structures whose boundary conditions become invertible to the policy sets recognition techniques was artificial neural photonics. Neuromorphic nanotechnology complies not only to a prototype of the biological system, but with today's manufacturing industrial systems, allowing its relatively close development [71].

$n m$

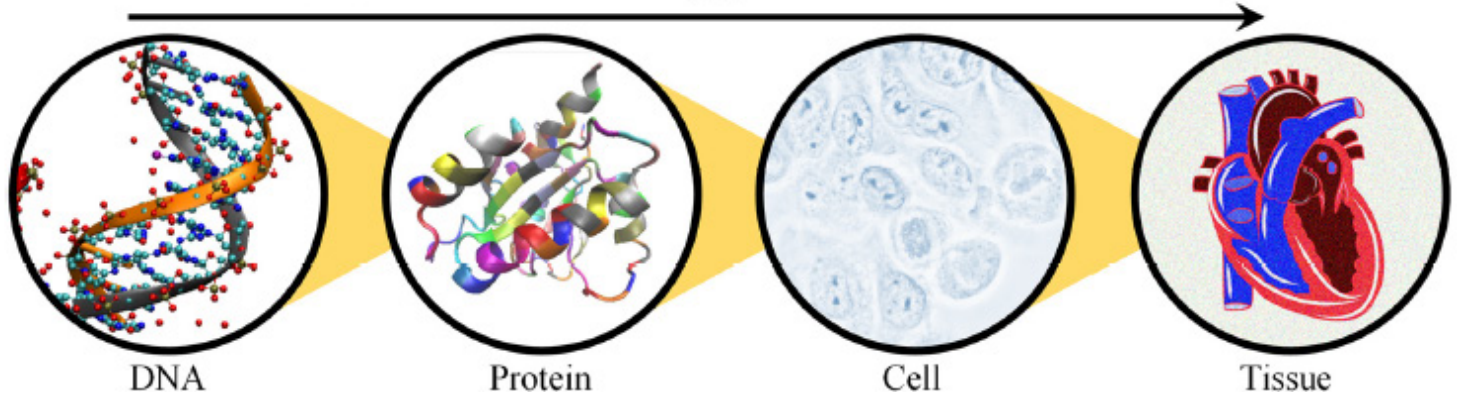

Figure 4 Structure of materials in life at different length scales [46].

Nano engineering allows microscale model modification of nanostructured materials as well as the creation of biomaterials containing controlled features. Its value of computational modelling would be that the Nano as well as microscale secrets concealed within substance output only at molecular level could be found. A multiscale review, comparative portrayal of nanomaterials through nanotechnology simulation to macroscopic scales measurement is seen in the above Figure 4. Design of life-time substances at spatial levels of duration. DNA inside the cell membrane only at microscale is indeed a large compound. DNA software segments of particular proteins that are normally mesoscale-modelled. Such substances operate throughout the molecules where a sample can be seen. Protein classes merge to form substances, although cells combine together before the nanoscale for structures that are essential for individual beings. Lack of reliable molecular structures, molecular simulations will simulate the motions as well as responses of particles. One such constitution requires a molecule course throughout the simulation technique, generating simultaneous iterations involving substance microstructure geometrical configuration or representing characteristics within environmental conditions [71]. 


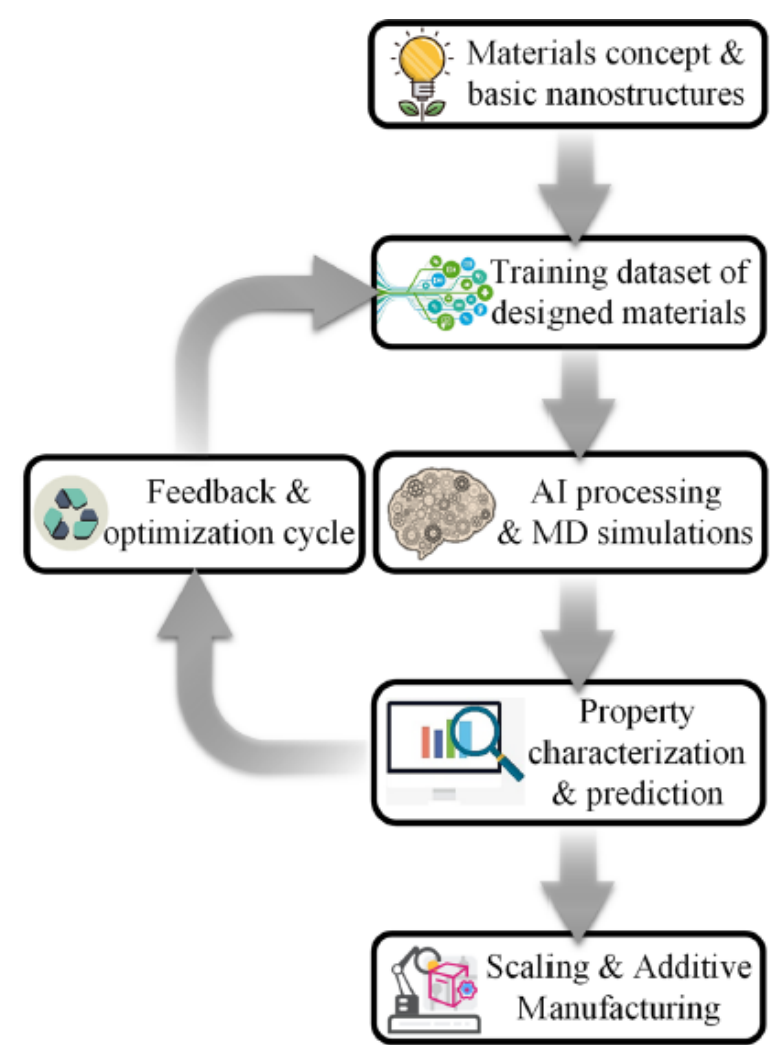

Figure 5 Flowchart of Nano engineering combined with AI for material modification and design [46]

The above Figure 5 summarizes a specific architecture schematic for computational modelling using AI. Although neural networks facilitate structural elements, through flexibility in types, designs, including quantities, additive manufacturing will speed up product development. In membrane patientspecific biomedical applications, heparin designed and synthesized protein gels assisted by additive manufacturing biomaterials substrates were produced as body epithelial simulation computations of osteogenic capability. Nanoparticles of performance and durability as well as the required to maintain human tissue has been shown to enable 3D biomaterials throughout the potential for tissue engineering. Increasing advanced development of nanomaterials with such a concept focused on AI is hypothesized to accelerate the invention of nanomaterials as well as diagnostic implants. A computational modelling schematic paired with AI including manipulating as well as modelling structures. This method primarily includes the steps below. The very $1^{\text {st }}$ challenge is to select the component idea through alteration or development, and also to acquire product technological characteristics. The collection of constructed substances would then be gathered through observations including calculations associated with the physical principle of volume control technique. To classify process parameters as well as model material activities, several AI methods are implemented through dynamic simulation. For more improvement, the model analysis will be replicated. Eventually, with potential uses, the optimum production concept is converted through new goods through composite materials. 


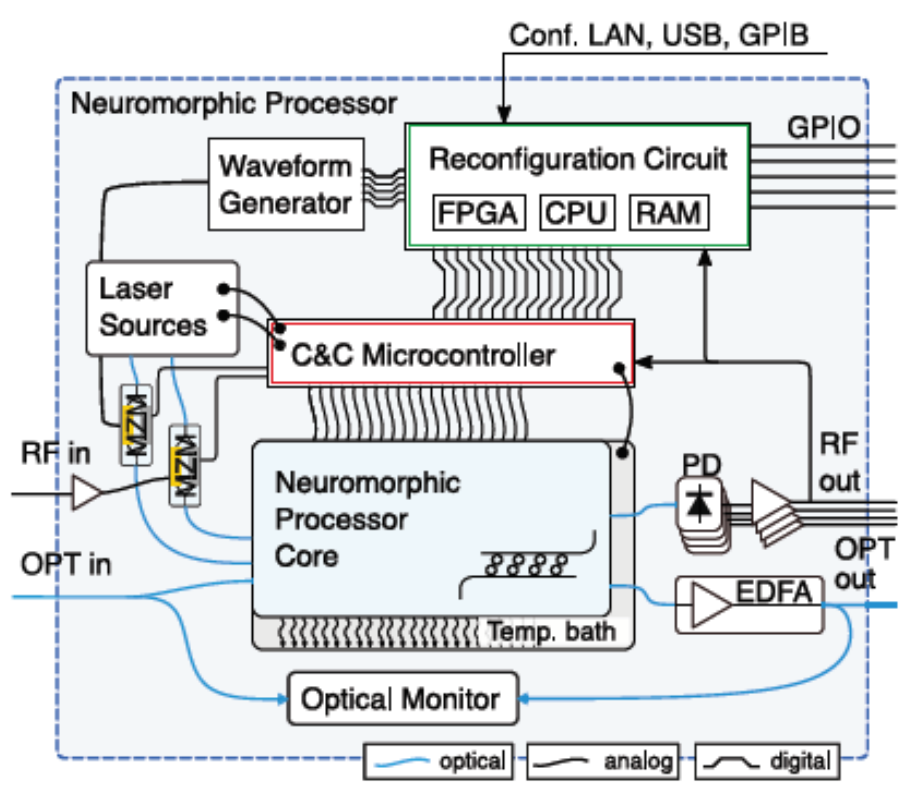

Figure 6 Simplified schematics of a neuromorphic processor [71].

The tunable superconducting computer model is used in the central processor, worthy of accepting a range of different empirical parameters, performing a variation, univariate transition, or generating storage facility outcomes. By default, a 'superconducting cell' is a system which can measure several transmitted signals, count them up, or calculate a probability distribution dependent mostly on count, in regards to providing the capacity to impulsive link simultaneously. As an alternative for visually different frequencies, this can specifically input through offset RF signals due to incorporated laser sources including photodiodes. The signal generation makes it possible to design random inputs which could be used as task in AI. Waveform is focused on some kind of potential connectivity framework bidirectional communication, whereby each contribution from each genetic algorithm, divided by wavelength, reside in such a common transducer. Compared to electrical integrated circuits, this needs to compensate for both the comparatively massive scale of superconducting devices. After all, a rational distinction among optoelectronic output measurements could only be made after combined scale including energy consumption of the whole computer system is counted, maybe not the capacity of MAC computing throughout the processing unit. Due to the production framework, the device PIC may have explicit or implicit laser beams or electromagnetic $\mathrm{I} / \mathrm{O}$ that cause additional costs when producing this high frequency source of light. Thermoelectric control and separation from many other temperature circuitries also are necessary that may have a detrimental impact mostly on processor's proper functioning. Enforced on a typical information system, the remote management circuitry adjusts the production deviations, controls the PIC toward inelastic scattering, and defends against the exposure to over-voltage. Figure 6 shows neuromorphic processor in [71].

Its key purpose is to transform a loss function into some kind of series of physical signal amplification liable for securing its mass structures throughout the PIC, electronically mounted to its operating storage. This does so through the synthesis of global laser configuration details, like spectrum or localized power density controllers and chip-embedded heating elements. They will essentially minimize mass locking's constant energy usage to a marginal amount relative to heater strategies. To monitor each and almost every mass module throughout the PIC, this micro-controller does have a quite large analog DC I/O list, as well as an elevated communication device with such a reconfiguration connection. The reconfiguration circuitry was its computer vision computer's greatest sub-processor and is also the reduced one. Associated with the input information from of the plant's detectors, the reconfiguration circuitry is being used to monitor the elevated switch. It collects CPU commands, living ecosystem information as well as the status of the management \& control circuit, which provides feedback as to how to customize the system in actual. The reconfiguration evaluation model the border between embedded computing designers as well as the superconducting developers. It therefore must choose someone who collects the guidance but keeps track not just to customize the quad core, and also to manage preparation, virtual education, including analog and digital interlinks. 


\section{Conclusions}

This paper overviews nanotechnology as a biomedical tool based on its applications and developments in the medical field where surface chemistry, physics and thermodynamics are used to determine toxic effect of nanomaterials. As metal oxide exhibit properties like significant bactericide behaviors, high surface area, improved magnetic and catalytic properties, so commonly used in the biomedical applications as nanoparticles. The target interference has the adverse effect on the photographs, leading to less precision. Dermoscopic is used as therapeutic imaging method as it is reliable and cost-effective. By test period elimination analysis, expense decreases and cluster analysis technique is used to decrease the test event volume. The levy method is employed in representation of test scenarios in cuckoo hunt. Futuristic structures help in innovative manufacturing, where structural implementation evaluation with proper characters and quality has high impact on the futuristic structures creation. The effective feature guidance, and molecular range favorable characteristics plays a major role in biodegradable polymer manipulation. The nanotechnology integrated with AI is drastically growing in biomaterials but quantity, reasonable quality management and strategies should be highly concentrated while introducing AI in biomedical field.

The frontend of electromagnetic should be changed to use advanced sophisticated multi antenna techniques and millimeter-wave channels. The neuromorphic nanotechnology is developing with today's manufacturing industrial systems.

\section{References}

[1] W Zhang, B Gao, J Tang, P Yao, S Yu, MF Chang, HJ Yoo, H Qian and H Wu. Neuro-inspired computing chips. Nat. Electron. 2020; 3, 371-82.

[2] L Calvet, JD Armas, D Masip and AA Juan. Learnheuristics: Hybridizing metaheuristics with machine learning for optimization with dynamic inputs. Open Math. 2017; 15, 261-80.

[3] I Boussaid, J Lepagnot and P Siarry. A survey on optimization metaheuristics. Inf. Sci. 2013; 237, 82-117.

[4] M Dorigo. 1992, Optimization, learning and natural algorithms. Dissertation. Politecnico di Milano, Italy.

[5] JD Farmer, NH Packard and AS Perelson. The immune system, adaptation, and machine learning, Phys. D Nonlinear Phenom. 1986; 22,187-204.

[6] JH Holland. Outline for a logical theory of adaptive systems. J. ACM 1962; 9, 297-314.

[7] TA Feo and MGC Resende. A probabilistic heuristic for a computationally difficult set covering problem. Oper. Res. Lett. 1989; 8, 67-71.

[8] O Martin, SW Otto and EW Felten. Large-step markov chains for the TSP incorporating local search heuristics. Oper. Res. Lett. 1992; 11, 219-24.

[9] J Kennedy and RC Eberhart. Particle swarm optimization. In: Proceedings of the IEEE International Conference on Neural Networks, Perth, WA, Australia. 1995, p. 1942-8.

[10] F Glover. Heuristics for integer programming using surrogate constraints. Decis. Sci. 1977; 8, 15666.

[11] S Kirkpatrick. Optimization by simulated annealing: Quantitative studies. J. Stat. phys. 1984; 34, 975-86.

[12] F Glover. Future paths for integer programming and links to artificial intelligence. Comput. Oper. Res. 1986; 13, 533-49.

[13] N Mladenovic. A variable neighborhood algorithm: A new metaheuristic for combinatorial optimization. Presented at Optimization Days, Montreal, 1995, p. 112.

[14] VS Saji, HC Choe and KWK Young. Nanotechnology in biomedical applications-a review. Int. J. Nano Biomater. 2010; 3, 119-39.

[15] AK Gupta, RR Naregalkar, VD Vaidya and M Gupta. Recent advances on surface engineering of magnetic iron oxide nanoparticles and their biomedical applications. Nanomedicine 2007; 2, 23-39.

[16] B Kasemo. Biological surface science. Surf. Sci. 2002; 500, 656-77.

[17] AH Faraji and P Wipf. Nanoparticles in cellular drug delivery. Bioorg. Med. Chem. 2009; 17, 295062.

[18] LE McNamara, RJ McMurray, MJP Biggs, F Kantawong, ROC Oreffo and MJ Dalby. Nanotopographical control of stem cell differentiation. J Tissue Eng. 2010; 2010, 120623.

[19] EKF Yim, EM Darling, K Kulangara, F Guilak and KW Leong. Nanotopography-induced changes in focal adhesions, cytoskeletal organization, and mechanical properties of human mesenchymal stem cells. Biomaterials 2010; 31, 1299-306. 
[20] DG Castner and BD Ratner. Biomedical surface science: Foundations to frontiers. Surf. Sci. 2002; 500, 28-60.

[21] DF Moyano and VM Rotello. Nano meets biology: Structure and function at the nanoparticle interface. Langmuir 2011; 27, 10376-85.

[22] KL Menzies and L Jones. The impact of contact angle on the biocompatibility of biomaterials. Optom. Vis. Sci. 2010; 87, 387-99.

[23] J Oh, MD Feldman, J Kim, C Condit, S Emelianov and TE Milner. Detection of magnetic nanoparticles in tissue using magneto-motive ultrasound. Nanotechnology 2006; 17, 4183-90.

[24] AK Gupta and M Gupta. Synthesis and surface engineering of iron oxide nanoparticles for biomedical applications. Biomaterials 2005; 26, 3995-4021.

[25] H Meng, Z Chen, G Xing, H Yuan, C Chen, F Zhao, C Zhang and Y Zhao. Ultrahigh reactivity provokes nanotoxicity: Explanation of oral toxicity of nano-copper particles. Toxicol. Lett. 2007; 175, 102-10.

[26] K Donaldson and A Seaton. The Janus faces of nanoparticles. J. Nanosci. Nanotechnol. 2007; 7, 4607-11.

[27] S Andreescu, M Ornatska, JS Erlichman, A Estevez and JC Leiter. Biomedical applications of metal oxide nanoparticles. In: E Matijevic (Ed.). Fine particles in medicine and pharmacy. Springer, Boston, MA, 2012.

[28] CT Campbell and CHF Peden. Oxygen vacancies and catalysis on ceria surfaces. Science 2005; 309, 713-4.

[29] AH Faraji and P Wipf. Nanoparticles in cellular drug delivery. Bioorg. Med. Chem. 2009; 17, 295062.

[30] HT Song, JS Choi, YM Huh, S Kim, YW Jun, JS Suh and J Cheon. Surface modulation of magnetic nanocrystals in the development of highly efficient magnetic resonance probes for intracellular labeling. J. Am. Chem. Soc. 2005; 127, 9992-3.

[31] NL Rosi and CA Mirkin. Nanostructures in biodiagnostics. Chem. Rev. 2005; 105, 1547-62.

[32] Z Xu, FR Sheykhahmad, N Ghadimi and N Razmjooy. Computer-aided diagnosis of skin cancer based on soft computing techniques. Open Med. 2020; 15, 860-71.

[33] P Moallem and N Razmjooy. A multi layer perceptron neural network trained by invasive weed optimization for potato color image segmentation. Trends Appl. Sci. Res. 2012; 7, 445-55.

[34] H Leng, X Li, J Zhu, H Tang, Z Zhang and N Ghadimi. A new wind power prediction method based on ridgelet transforms, hybrid feature selection and closed-loop forecasting. Adv. Eng. Inform. 2018; 36, 20-30.

[35] A Hekler, JS Utikal, AH Enk, A Hauschild, M Weichenthal, RC Maron, C Berking, S Haferkamp, J Klode, D Schadendorf, B Schilling, T Holland-Letz, B Izar, CV Kalle, S Frohling and TJ Brinker. Superior skin cancer classification by the combination of human and artificial intelligence. Eur. J. Cancer 2019; 120, 114-21.

[36] D Anoraganingrum. Cell segmentation with median filter and mathematical morphology operation. In: Proceedings of the $10^{\text {th }}$ International Conference on Image Analysis and Processing, Venice, Italy. 1999, p. 1043-6.

[37] T Loupas, WN McDicken and PL Allan. An adaptive weighted median filter for speckle suppression in medical ultrasonic images. IEEE Trans. Circuits Syst. 1989; 36, 129-35.

[38] E Emary, HM Zawbaa and C Grosan. Experienced gray wolfoptimization through reinforcement learning and neural networks. IEEE Trans. Neural Netw. Learn. Syst. 2018; 29, 681-94.

[39] I Giotis, N Molders, S Land, M Biehl, MF Jonkman and N Petkov. MED-NODE: A computerassisted melanoma diagnosis system using non-dermoscopic images. Expert Syst. Appl. 2015; 42, 6578-85.

[40] SHS Moosavi and VK Bardsiri. Satin bowerbird optimizer: A new optimization algorithm to optimize ANFIS for software development effort estimation. Eng. Appl. Artif. Intell. 2017; 60, 1-15.

[41] P Lakshminarayana and TV SureshKumar. Automatic generation and optimization of test case using hybrid cuckoo search and bee colony algorithm. J. Intell. Syst. 2020; 30, 59-72.

[42] SK Dubey and B Jasra. Reliability assessment of component based software systems using fuzzy and ANFIS techniques. Int. J. Syst. Assur. Eng. Manag. 2017; 8, 1319-26.

[43] T Jie, Z Yong and W Lina. Neural network based software reliability prediction with the feed of testing process knowledge. In: Proceedings of the International Conference on Information Technology and Software Engineering, Beijing. 2012, p. 19-27.

[44] A Kaushik, S Verma, HJ Singh and G Chhabra. Software cost optimization integrating fuzzy system and COA-Cuckoo optimization algorithm. Int. J. Syst. Assur. Eng. Manag. 2017; 8, 1461-71. 
[45] A Zaryabi and AB Hamza. A neural network approach for optimal software testing and maintenance. Neural Comput. Appl. 2014; 24, 453-61.

[46] W Jian, D Hui and D Lau. Nanoengineering in biomedicine: Current development and future perspectives. Nanotechnol. Rev. 2020; 9, 700-15.

[47] D Kim, K Shin, SG Kwon and T Hyeon. Synthesis and biomedical applications of multifunctional nanoparticles. Adv. Mater. 2018; 30, 1802309.

[48] Q Yan, H Dong, J Su, J Han, B Song, Q Wei and Y Shi. A review of 3D printing technology for medical applications. Engineering 2018; 4, 729-42.

[49] J Li, Y Liu, J Ren, BZ Tay, T Luo, L Fan, D Sun, G Luo, D Lau, Marcos and RHW Lam. Antibodycoated microstructures for selective isolation of immune cells in blood. Lab Chip 2020; 20, 107282.

[50] M Karplus and JA McCammon. Molecular dynamics simulations of biomolecules. Nat. Struct. Biol. 2002; 9, 646-52.

[51] MJ Buehler. Atomistic modeling of materials failure. Springer Science \& Business Media, New York, 2008.

[52] S Ramakrishna, J Mayer, E Wintermantel and KW Leong. Biomedical applications of polymercomposite materials: A review. Compos. Sci. Technol. 2001; 61, 1189-224.

[53] M Niinomi. Recent metallic materials for biomedical applications. Metall. Mater. Trans. A 2002; 33, 477.

[54] MW Tibbitt, CB Rodell, JA Burdick and KS Anseth. Progress in material design for biomedical applications. Proc. Natl. Acad. Sci. USA 2015; 112, 14444-51.

[55] PK Jain, X Huang, IH El-Sayed and MA El-Sayed. Noble metals on the nanoscale: Optical and photothermal properties and some applications in imaging, sensing, biology, and medicine. Acc. Chem. Res. 2008; 41, 1578-86.

[56] S Pardeep. NiTi shape memory alloy: Physical and tribological characterization. J. Mech. Behav. Mater. 2018; 27, 20180009.

[57] AS Bale, SV Reddy and SA Huddar. Electromechanical characterization of Nitinol based RF MEMS switch. Mater. Today Proc. 2020; 27, 443-5.

[58] AS Bale, SV Reddy and S Tiwari. Effect of residual stress on resonant frequency in Nitinol based thin film resonator. IOP Conf. Ser. Mater. Sci. Eng. 2020; 872, 012008.

[59] SA Huddar, BG Sheeparamatti and AS Bale. Study of pull-in voltage of a perforated SMA based MEMS Switch. In: Proceedings of the International Conference on Microelectronic Devices, Circuits and Systems, Vellore, India. 2017, p. 1-4.

[60] E Okur and M Turkan. A survey on automated melanoma detection. Eng. Appl. Artif. Intell. 2018; 73, 50-67.

[61] Melanoma.Org, Melanoma facts and statistics, Available at: https://www.melanoma.org.au /understanding-melanoma/melanoma-facts-and-statistics/, accessed July 2017.

[62] Medicine Net, What is melanoma?, Available at: http://www.medicinenet.com/melanoma/ article.htmwhat-is-melanoma, accessed July 2017.

[63] PDQ Adult Treatment Editorial Board, PDQ Melanoma treatment, Available at: https:// www.cancer.gov/types/skin/patient/melanoma-treatment-pdq, accessed February 2018.

[64] PDQ Adult Treatment Editorial Board, Available at: https://www.cancer.gov/publications/pdq/ editorial-boards/adult-treatment, accessed December 2018.

[65] AD Katsambas, TM Lotti, C Dessinioti and AM D'Erme. European handbook of dermatological treatments. Springer, 2015.

[66] E Senel. Dermatoscopy of non-melanocytic skin tumors. Indian J. Dermatol. Venereol. Leprol. 2011; 77, 16-21.

[67] MA Msrtinez-del-Amor, I Perez-Hurtado, D Orellana-Martin and MJ Perez-Jimenez. Adaptative parallel simulators for bioinspired computing models. Future Gener. Comput. Syst. 2020; 107, 46984.

[68] G Paun. Computing with membranes. J. Comput. Syst. Sci. 2000; 61, 108-43

[69] D Diaz-Pernil, C Graciani-Diaz, MA Gutierrez-Naranjo, I Perez-Hurtado and MJ Perez-Jimenez. Software for P systems. In: G Paun, G Rozenberg and A Salomaa (Eds.). The Oxford handbook of membrane computing. Oxford University Press, Oxford, UK, 2010, p. 437-54.

[70] J Blakes, J Twycross, FJ Romero-Campero and N Krasnogor. The Infobiotics Workbench: An integrated in silico modelling platform for Systems and Synthetic Biology. Bioinformatics 2011; 27, $3323-4$ 
[71] TFD Lima, AN Tait, A Mehrabian, A Mehrabian, MA Nahmias, C Huang, HT Peng, BA Marquez, M Miscuglio, T El-Ghazawi, VJ Sorger, BJ Shastri and PR Prucnal. Primer on silicon neuromorphic photonic processors: Architecture and compiler. Nanophotonics 2020; 9, 4055-73.

[72] T Rudolph. Why I am optimistic about the silicon-photonic route to quantum computing. $A P L$ Photonics 2017; 2, 030901.

[73] JW Silverstone, D Bonneau, JL O’Brien and MG Thompson. Silicon quantum photonics. IEEE J. Sel. Top. Quantum Electron. 2016; 22, 390-402.

[74] D Brunner, MC Soriano, CR Mirasso and I Fischer. Parallel photonic information processing at gigabyte per second data rates using transient states. Nat. Commun. 2013; 4, 1364.

[75] K Vandoorne, P Mechet, TV Vaerenbergh, M Fiers, G Morthier, D Verstraeten, B Schrauwen, J Dambre and P Bienstman. Experimental demonstration of reservoir computing on a silicon photonics chip. Nat. Commun. 2014; 5, 3541.

[76] MC Soriano, D Brunner, M Escalona-Moran, CR Mirasso and I Fischer. Minimal approach to neuro-inspired information processing. Front. Comput. Neurosci. 2015; 9, 68

[77] F Duport, A Smerieri, A Akrout, M Haelterman and S Massar. Fully analogue photonic reservoir computer. Sci. Rep. 2016; 6, 22381. 